\title{
Financial Crisis 2007-2009. How Real Estate Bubble and Transparency and Accountability Issues Generated and Worsen the Crisis \\ Bilal Aziz*
}

\begin{abstract}
This paper seeks to explain some main factors behind the Financial Crisis 20072009 with a special focus on the Real Estate Bubble and Transparency and Accountability Issues in US Financial System and how these two factors generated and worsen the crisis. Financial Crisis 2007-2009, which starts from the United States sub-prime mortgage market and spread to US financial sector and later on spread to the rest of world, is said to be an even bigger crisis than the Great Depression of 1929. This crisis is unique in this way and we haven't seen such a bigger impact world wide from any other crisis. This paper would empirically prove the main causes which are right in the heart of the crisis and least discussed.
\end{abstract}

Keywords: sub-prime mortgages, housing bubble, mortgage back securities, conduits, wealth and income inequalities.

\begin{abstract}
Resumen
Este trabajo busca explicar algunos de los factores principales detrás de la crisis financiera de 2007-2009, con especial énfasis en la burbuja inmobiliaria, en la rendición de cuentas en el sistema financiero de Estados Unidos y en cómo estos dos factores generaron y empeoraron la crisis. La crisis financiera 2007-2009 inició con las hipotecas de alto riesgo del mercado inmobiliario, se extendió al sector financiero y, finalmente, al resto del mundo. Se dice que fue una crisis aun más grande que la Gran Depresión de 1929 y se le califica como única toda vez que no se conoce otra que haya impactado al mundo de esta manera. Este trabajo empírico pretende demostrar las causas que están en el corazón de la crisis y no han sido lo suficientemente discutidas.
\end{abstract}

Palabras clave: hipotecas sub-prime, burbuja inmobiliaria, títulos respaldados por hipotecas, conductos, riqueza y desigualdad de ingresos.

\footnotetext{
* Department of Economics, University of the Punjab, Pakistan. E mail: bilalazizposwal@hotmail.com
} 


\section{Introduction}

Financial Crises are the result of the normal functioning of the economic and financial systems over the course of the business cycle. Endogenous processes take place near the peak of the expansion phase of the business cycle, in particular, the deterioration of the financial condition of the business sector, which set the stage for a financial crisis (Wolfson, 1994). There is no precise definition of "financial crisis", but a common view is that disruptions in financial markets rise to the level of a crisis when the flow of credit to households and businesses is constrained and the real economy of goods and services is adversely affected. One thing is common in all crises: that "All Crises are Crises of Success" (Portes and Vines, 1997).

The current tsunami in financial markets, which is believed to have been triggered by the collapse of the sub-prime housing market, has refocused the ideas of Hyman Minsky (1919-1996), a prominent member of the post-Keynesian school of economics. Many commentators are of the view that Minsky accurately anticipated the current financial crisis (Wray, 2008; McCauley, 2008). Some of them called this situation a "Minsky moment" (Whalen, 2007; Magnus, 2007). He is described as the "obscure economist" who identified highly speculative "Ponzi Finance" as an underlying factor in such crises. But identifying Ponzi finance is not the most important contribution Minsky has made to our understanding of the logic of repeated financial crises under capitalism (Kregel, 2008). Minsky says in his book Stabilizing the instable economy: "The Economic instability so evident since the late 1960s is the result of the fragile financial system that emerged from cumulative changes in the financial relations and institutions over the years following World War II" (Minsky, 1986).

While some mainstream economists are of the view that economic busts are the outcome of various external shocks to the economy and regulatory flaws on the part of Federal Reserve (Shostak, 2008) and Government actions (Taylor, 2008), Minsky held that, even in the absence of such shocks, the capitalistic economy has an inherent tendency to develop instability, which culminates in severe economic crises. The key mechanism that pushes the economy towards a crisis is the Economic System, which is not natural. Minsky says: "Economic Systems are not natural systems. An economy is a social organization created either through legislation or 
by an evolutionary process of invention and innovation" (Minsky, 1986). The heart of Minsk's framework is Financial Instability Hypothesis (FIH), which says that capitalism is inherently unstable and has self-destructive tendencies.

Another aspect of the Financial Instability Hypothesis is that, during good times, banks and other intermediaries strive to innovate with regard to the assets they acquire and the liabilities they market. This means that, during good times, financial intermediaries (Minsky labeled them as "merchants of debts") try to lure investors to buy the debt by means of sophisticated innovations. The chase for making more profits causes players in financial markets to place their money in various investments that have very little substance — such as sub prime-mortgage-backed securities. What makes these investments attractive is sophisticated packaging and the relatively high rate of return. But, once economic conditions change, the true state of many borrowers comes to the surface and leads to a crisis. Lenders curtail their supply of funds and borrowers are pushed to bankruptcy, for they cannot renew their borrowing to pay debts - a financial crisis emerges.

Apart from the introduction the paper has been divided into three main parts. First we would discuss the Real Estate Market boom bubble and bust, and secondly the lack of transparency and accountability issues would be discussed. Finally, after some empirical analysis, we would draw some conclusions

But before going forward let's have a look at the present situation at the US economy. I have collected thirty years data along with twenty two variables to make a comparison of the economy. Last decade was a devastating for the US, this is largely due to two wars which they fought in Afghanistan and Iraq. This data shows a very trouble decade ahead for the US economy.

Specially the sky high Govt. Debts, widening Current Account deficit and ever increased unemployment rate are very dangerous signs for the future economy. As shown in the data, that appreciation of house prices were in negative along with the every decreasing interest rate, which were the main cause of subprime default. 


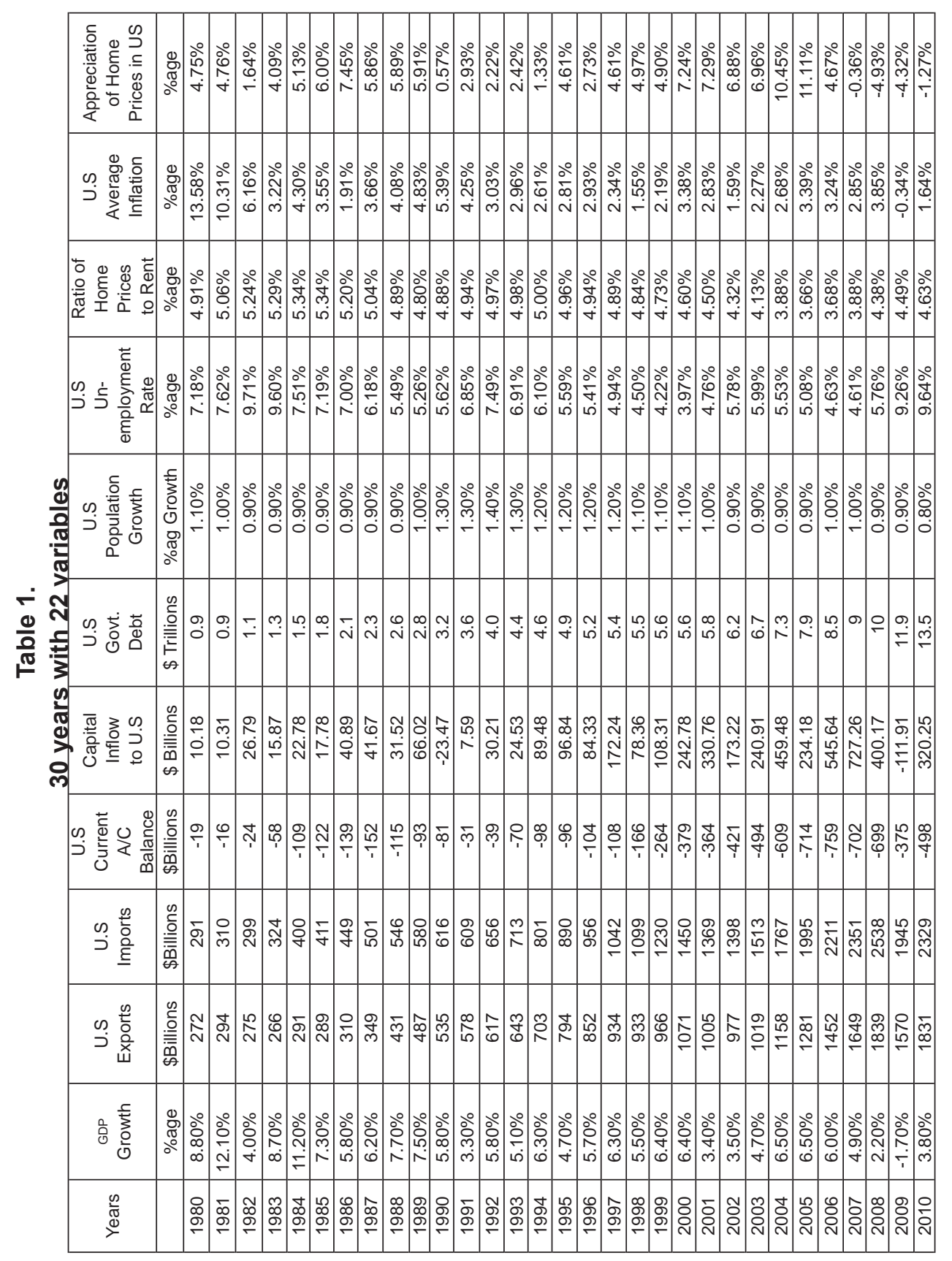




\begin{tabular}{|c|c|c|c|c|c|c|c|c|c|c|c|c|c|c|c|c|c|c|c|c|c|c|c|c|c|c|c|}
\hline 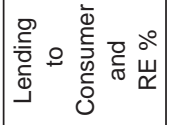 & $\left|\begin{array}{c}0 \\
\mathbb{8} \\
0 \\
0 \\
0\end{array}\right|$ & ஓें & $\mid \begin{array}{l}0 \\
0 \\
0 \\
0\end{array}$ & 总 & ْे & 总 & 离 & 今े & ஓें & 号 & ஓें & ஓ̊ำ & 通 & 过 & ঐे & ণั & 咢 & 令 & 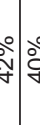 & $\frac{\circ}{8}$ & & 今̊ & & & $\begin{array}{l}\circ \\
f\end{array}$ & ஓें & 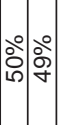 \\
\hline 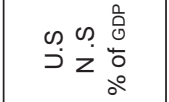 & \begin{tabular}{|c|}
0 \\
0 \\
$\mathbb{\pi}$ \\
0 \\
0
\end{tabular} & 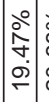 & 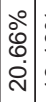 & 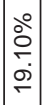 & $\begin{array}{l}\stackrel{\circ}{\circ} \\
\dot{0} \\
\end{array}$ & 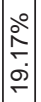 & 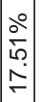 & 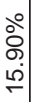 & 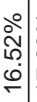 & 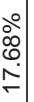 & $\begin{array}{l}0 \\
\stackrel{0}{0} \\
0 \\
\dot{0} \\
0\end{array}$ & 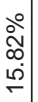 & 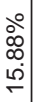 & 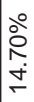 & 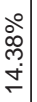 & 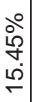 & . & مَّ. & 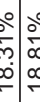 & 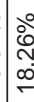 & $\begin{array}{l}\stackrel{0}{\circ} \\
\varnothing \\
\infty\end{array}$ & $\mid \begin{array}{c}0 \\
\vdots \\
\sigma \\
6 \\
ஸ\end{array}$ & & & 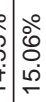 & 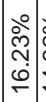 & 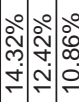 \\
\hline$\overline{\mathrm{Z}}$ & 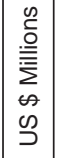 & 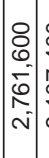 & $\left|\begin{array}{c}8 \\
\vdots \\
\vdots \\
0 \\
\hdashline \\
\end{array}\right|$ & $\mid \begin{array}{l}8 \\
2 \\
10 \\
0 \\
0 \\
n \\
m\end{array}$ & $\begin{array}{l}\text { O } \\
\stackrel{N}{1} \\
8 \\
8 \\
0 \\
\text { m. }\end{array}$ & 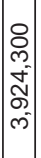 & 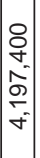 & 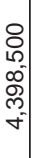 & $\begin{array}{l}8 \\
0 \\
\\
0 \\
0 \\
0 \\
+\end{array}$ & $\begin{array}{l}8 \\
0 \\
\hat{0} \\
0 \\
\frac{0}{\sigma^{\circ}}\end{array}$ & 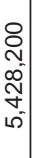 & 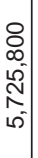 & 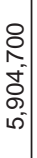 & $\begin{array}{l}8 \\
1 \\
m \\
\\
0 \\
0\end{array}$ & 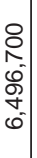 & 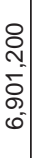 & 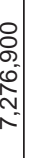 & 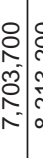 & 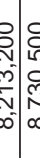 & $\mid \begin{array}{l}8 \\
2 \\
2 \\
\infty \\
\\
0\end{array}$ & $\begin{array}{l}8 \\
0 \\
0 \\
0 \\
0 \\
\text { o }\end{array}$ & $\begin{array}{c}2 \\
N \\
0 \\
0 \\
0 \\
0 \\
-1\end{array}$ & & & 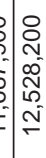 & & 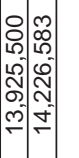 \\
\hline 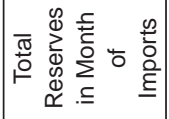 & $\begin{array}{l}\stackrel{\infty}{ \pm} \\
\stackrel{ \pm}{ \pm} \\
\stackrel{0}{\Sigma}\end{array}$ & $\mid \begin{array}{c}\sim \\
0 \\
0\end{array}$ & $\underset{f}{\sigma}$ & $\begin{array}{l}\infty \\
\dot{\sim}\end{array}$ & $\begin{array}{l}\sigma \\
\text { ले }\end{array}$ & $\mid \hat{\mathrm{N}}$ & $\mid \begin{array}{r}\text { S } \\
\end{array}$ & & & & $\begin{array}{c}\infty \\
\stackrel{N}{N}\end{array}$ & $\hat{\mathrm{N}}$ & $\begin{array}{l}\stackrel{\varphi}{\mathrm{N}} \\
\text {. }\end{array}$ & $\stackrel{m}{\sim}$ & $\stackrel{\vec{v}}{\mathrm{i}}$ & $\overline{\mathrm{i}}$ & $\sim$ & & בת & $=$ & o̊. & - & & & $\because \mathscr{0}$ & $\mid \begin{array}{l}0 \\
0 \\
0\end{array}$ & $\because ت$ \\
\hline 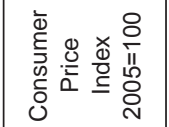 & 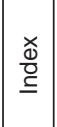 & 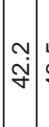 & $\left|\begin{array}{l}1 \\
0 \\
\dot{\theta}\end{array}\right|$ & $\begin{array}{l}\dot{S} \\
\dot{\sigma}\end{array}$ & 5 & 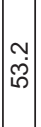 & 它 & $\begin{array}{c}- \\
\dot{6} \\
\end{array}$ & 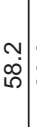 & $\begin{array}{l}0 \\
\vdots \\
0\end{array}$ & 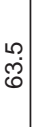 & $\begin{array}{l}\dot{\theta} \\
\dot{\theta}\end{array}$ & $\hat{8}$ & $\frac{\infty}{\Gamma}$ & $\stackrel{\sim}{\sim}$ & $\begin{array}{l}\stackrel{\sigma}{\rho} \\
\stackrel{\rho}{\rho}\end{array}$ & $\infty$ & 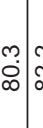 & 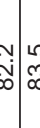 & $\begin{array}{l}m \\
\infty \\
\infty \\
\infty\end{array}$ & $\begin{array}{l}\infty \\
\infty \\
\infty \\
\infty\end{array}$ & 今ं & নু் & & 음 & & 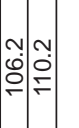 \\
\hline 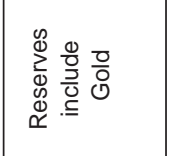 & 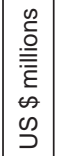 & 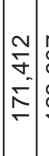 & 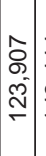 & 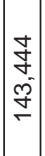 & 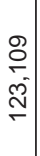 & 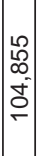 & $\begin{array}{c}\infty \\
\infty \\
\infty \\
N \\
=\end{array}$ & $\begin{array}{l}m \\
\infty \\
\infty \\
\infty \\
m \\
- \\
-1\end{array}$ & $\mid$ & 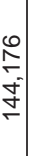 & $\mid \begin{array}{l}\infty \\
\infty \\
0 \\
\infty \\
\infty \\
0 \\
0\end{array}$ & $\begin{array}{l}\mathscr{D} \\
0 \\
0 \\
\\
\end{array}$ & 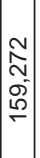 & 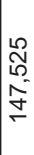 & 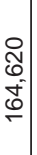 & 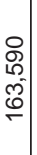 & 足 & $\begin{array}{l}8 \\
8 \\
8\end{array}$ & 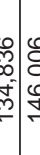 & 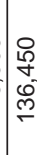 & 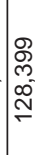 & $\begin{array}{l}0 \\
0 \\
0 \\
0 \\
0 \\
-1\end{array}$ & & & 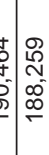 & $\mid$ & 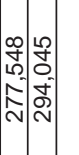 \\
\hline 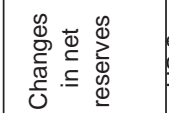 & 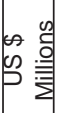 & & $\mid$\begin{tabular}{l}
$\infty$ \\
0 \\
0 \\
\hdashline \\
1 \\
1
\end{tabular} & $\begin{array}{l}\infty \\
\stackrel{0}{0} \\
\dot{7} \\
1\end{array}$ & $\begin{array}{l}\mathscr{8} \\
\frac{5}{-} \\
\end{array}$ & 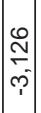 & $\mid$ & 을 & $\begin{array}{l}\hat{f} \\
\bar{\sigma}\end{array}$ & 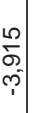 & 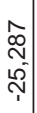 & $\mid$ & & $\begin{array}{l}\hat{N} \\
\text { ले }\end{array}$ & $\left|\begin{array}{l}0 \\
0 \\
0 \\
\\
\end{array}\right|$ & 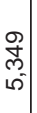 & 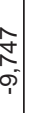 & $\begin{array}{l}8 \\
\varnothing \\
6\end{array}$ & 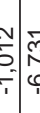 & & & $\begin{array}{l}0 \\
\tilde{W} \\
o \\
\forall \\
i\end{array}$ & 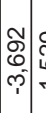 & & 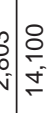 & & 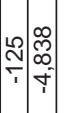 \\
\hline $\begin{array}{l}\frac{0}{0} \\
\frac{0}{0} \\
\stackrel{0}{*} \\
\stackrel{0}{0} \\
0\end{array}$ & 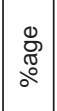 & $\begin{array}{l}0 \\
\circ \\
0 \\
\infty \\
\infty \\
2\end{array}$ & $\mid \begin{array}{c}0 \\
\circ \\
0 \\
\vdots \\
\grave{N}\end{array}$ & $\begin{array}{l}\stackrel{0}{\circ} \\
\stackrel{2}{ } \\
\infty \\
-\infty\end{array}$ & $\begin{array}{l}\circ \\
\stackrel{\rho}{0} \\
\stackrel{\rho}{\leftarrow}\end{array}$ & $\mid$\begin{tabular}{c}
0 \\
$\vdots$ \\
\hdashline \\
$\infty$ \\
\hdashline
\end{tabular} & 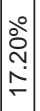 & 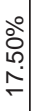 & $\begin{array}{l}0 \\
0 \\
0 \\
0 \\
\infty \\
-\end{array}$ & 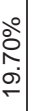 & 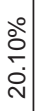 & 隹 & 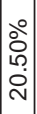 & 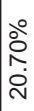 & 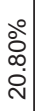 & 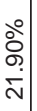 & 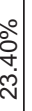 & 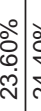 & 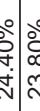 & 总 & & 今̊ & 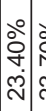 & & 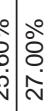 & 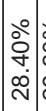 & \\
\hline 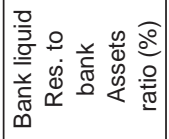 & 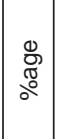 & 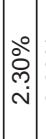 & $\mid \begin{array}{l}0 \\
\stackrel{\circ}{0} \\
\text { N่ }\end{array}$ & 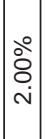 & 号 & 今̊ & & & 今̊. & $\begin{array}{c}0 \\
\stackrel{0}{0} \\
\infty \\
- \\
-\end{array}$ & 总 & & 官 & & 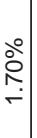 & ه̊ํ. & & r. & مإِ & ठ̊. & 。̊̊ & ○े & & & $\begin{array}{l}0 \\
0 \\
0 \\
0\end{array}$ & & \\
\hline 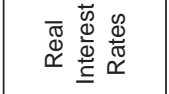 & 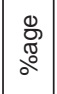 & 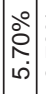 & $\mid \begin{array}{c}0 \\
0 \\
0 \\
\infty \\
\infty\end{array}$ & ○ें & $\begin{array}{l}\circ \\
\vdots \\
\vdots \\
\dot{0}\end{array}$ & $\mid \begin{array}{l}0 \\
8 \\
\varnothing \\
\infty\end{array}$ & $\begin{array}{c}0 \\
0 \\
0 \\
0 \\
0\end{array}$ & $\begin{array}{l}\text { o̊ } \\
\vdots \\
0 \\
\dot{0}\end{array}$ & $\begin{array}{l}\circ \\
\text { 今े } \\
\text { ले } \\
\llcorner\end{array}$ & 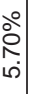 & $\begin{array}{l}0 \\
\vdots \\
0 \\
0 \\
0\end{array}$ & 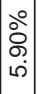 & $\begin{array}{l}\mid \\
\stackrel{0}{0} \\
0 \\
\infty \\
\dot{+}\end{array}$ & $\begin{array}{l}\circ \\
\text { Oे } \\
\\
\text { ஸे }\end{array}$ & $\begin{array}{l}0 \\
\circ \\
0 \\
0 \\
\dot{0}\end{array}$ & \begin{tabular}{|l|}
$\circ$ \\
oे \\
\\
$\dot{\sigma}$
\end{tabular} & مُ & 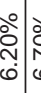 & 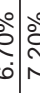 & 总 & & $\begin{array}{c}0 \\
\stackrel{0}{0} \\
\dot{+} \\
\dot{0}\end{array}$ & 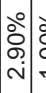 & & 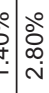 & & 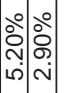 \\
\hline 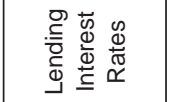 & 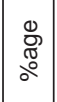 & 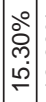 & . & $\begin{array}{l}\text { Oें } \\
\text { Oे. } \\
\dot{+}\end{array}$ & $\begin{array}{l}0 \\
0 \\
\infty \\
0 \\
0\end{array}$ & 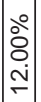 & & $\begin{array}{l}\text { ○े } \\
\text { مे } \\
\infty\end{array}$ & | & ○े| & 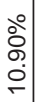 & $\begin{array}{l}0 \\
0 \\
0 \\
0 \\
0\end{array}$ & $\left|\begin{array}{l}\mid \\
0 \\
0 \\
0 \\
\infty \\
\infty\end{array}\right|$ & ○े & 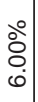 & 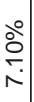 & $\begin{array}{l}\infty \\
\infty \\
\infty \\
\infty\end{array}$ & $\begin{array}{c}e_{0}^{\circ} \\
\infty \\
\infty\end{array}$ & 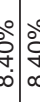 & & & ○े & ঃ̊ & & 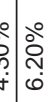 & & 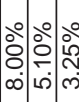 \\
\hline 岕 & & 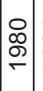 & $\begin{array}{l}- \\
\infty \\
\sigma \\
\sigma\end{array}$ & $\mid \begin{array}{c}\infty \\
\infty \\
\stackrel{\infty}{\sim}\end{array}$ & \begin{tabular}{l}
$\mathscr{D}$ \\
$\infty$ \\
\hdashline
\end{tabular} & 4 & & $\begin{array}{l}\mathscr{Q} \\
\stackrel{0}{ } \\
\stackrel{0}{-}\end{array}$ & 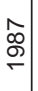 & $\begin{array}{c}\infty \\
\infty \\
\stackrel{8}{\circ} \\
\end{array}$ & 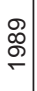 & $\begin{array}{l}\text { O } \\
\stackrel{\circ}{\circ} \\
\end{array}$ & 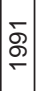 & \begin{tabular}{l}
\multirow{2}{*}{} \\
$\stackrel{-}{-}$
\end{tabular} & 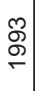 & |' & 8 & תু & S & \& & ठ্ণ & స్రి & 잉 & "ூ & ปे & 吕 & 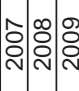 \\
\hline
\end{tabular}




\section{Housing Boom, Bubble \& Bust}

A housing bubble is a type of economic bubble that occurs periodically in local or global markets. It is characterized by rapid increases in valuation of real property such as housing until they reach unsustainable levels relative to income and other economic elements. The driving force behind the mortgage and financial market excesses that led to the current credit crisis was the sustained rise in house prices and the perception that they could go no where but up (Baily et al., 2008).

Figure 1 plots data on the ratio of the total value of residential real estate to a measure of the rental value at an annual rate. Equivalent to a price-earnings ratio for equity, data beginning in 1955 make clear how extraordinary the first five years of the $21^{\text {st }}$ century were. Normally, home prices are between 9 and 11 times the annual level of rent paid. That makes sense, as it implies an average user cost of housing of around $10 \%$. But since 2000, prices have skyrocketed, leaving rents in the dust. The price-to-rent ratio peaked at the end of 2006, reaching the rather extraordinary level of 14.5 , clearly suggesting the existence of a "bubble" in residential housing. Home prices were at levels far higher than justified by fundamental values (or replacement costs).

The residential real estate price rise that began in 2000 had a number of important side effects. First, when the value of housing rises, it creates wealth and wealthier people consume more. This consumption-wealth effect is substantial.

The simplest way to convert housing wealth into consumption is to borrow. And this is where, in hindsight, we can find the second sign of trouble. Figure 2 separates the value of residential housing into owners' equity and borrowing (combining mortgages and home equity loans). What we see is that as the value of residential real estate rose, mortgage borrowing increased even faster. Since 1995 home equity has fallen from $58 \%$, already far below the $69 \%$ level a decade earlier, to $52 \%$ of home value.

To recap, by the beginning of 2007 we can say:

1. Home prices were at unprecedented levels.

2. Home owners had more leverage than ever before.

3. Mortgage quality had declined substantially. 
Figure 1.

Ratio of home prices to rents

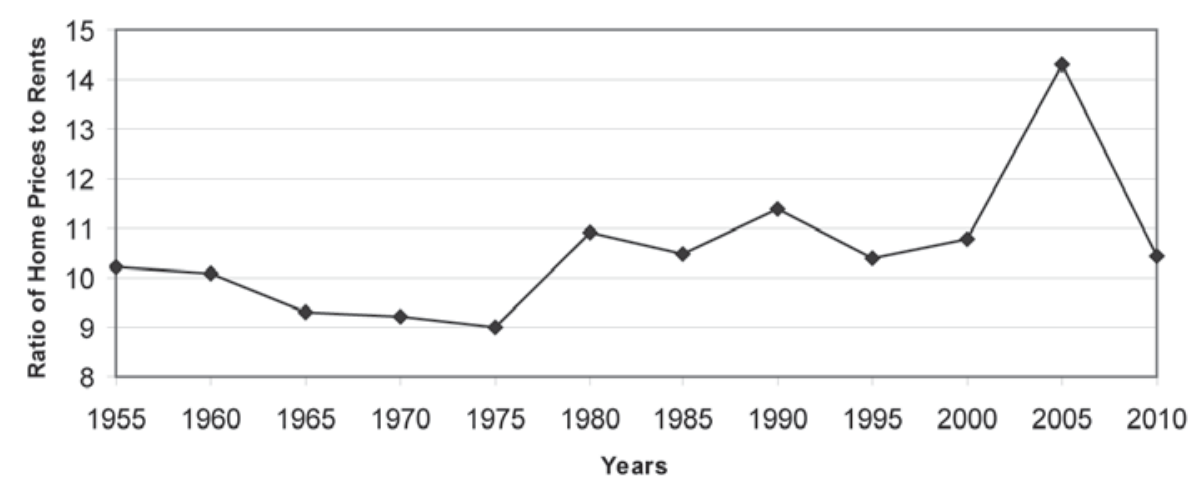

Source: Self-Made Figure (Data Source ofHeo).

Figure 2.

Evolution of Equity and Borrowing in Residential Real Estate

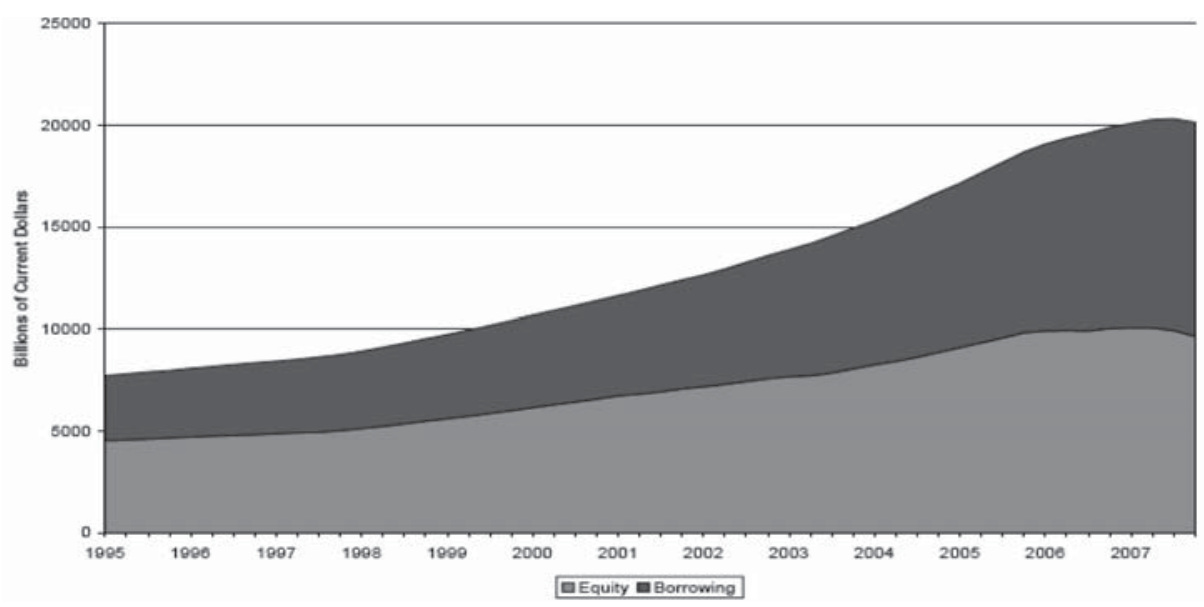

Source: Cecchetti, 2008. 
This sets the stage for the crisis (Cecchetti, 2008). House prices in some regions grew rapidly after interest rates declined in 2001. Adjusting for inflation, real U.S. house prices rose 34\% during 2000-2005 (they rose 51\% if not adjusted), which is more than double any five-year rate in the past 30 years. Specific regions experienced even faster appreciation; in 2004 alone, housing in Miami, Los Angeles, and West Palm Beach appreciated more than 20\% and Las Vegas appreciated 35\%. Figure 3 shows that the rate of house price appreciation, year over year, reached $13 \%$ in 2006, and then plunged to $3 \%$ by mid- 2007 .

A survey held by Case and Shiller (2003) report that the overwhelming majority of persons surveyed in 2003 agreed or strongly agreed with the statement that real estate is the best investment for long-term holders. Respondents expected prices to increase in the future at 6 to $15 \%$ a year, depending on location.

Figure 4 shows that, between 1975 and 1995 real home prices went through two cyclical waves: rising after 1975, falling in the early 1980s and then rising again before falling in the early 1990s. From 1975 until

Figure 3.

Appreciation of House Prices, 1996-2007

(percentage change year by year)

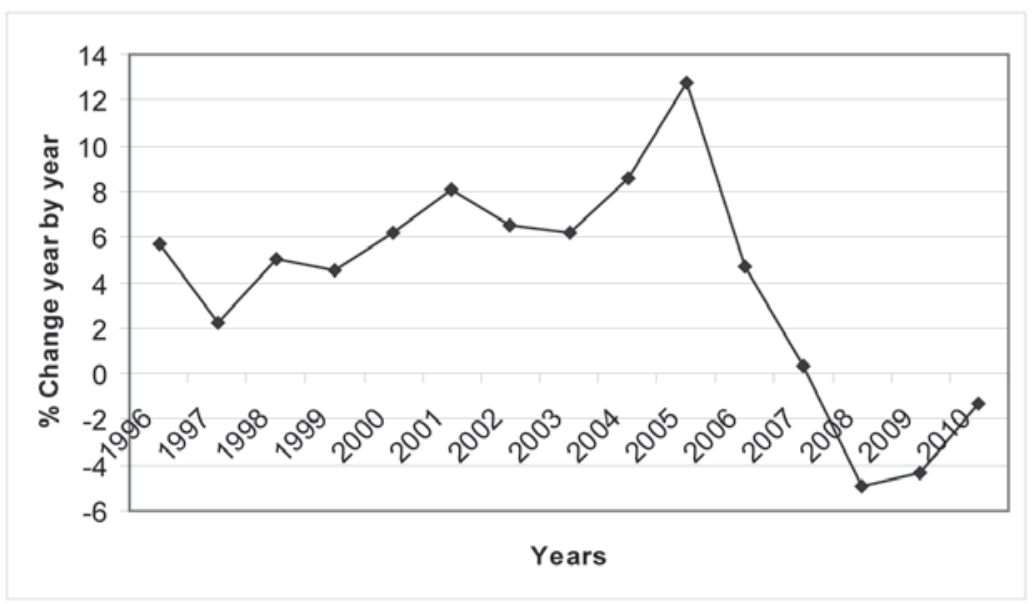

Source: Self-Made Figure (Data Source ofHeo). 
Figure 4.

Real home prices and real household income (1976=100); 30 -year conventional mortgage rate

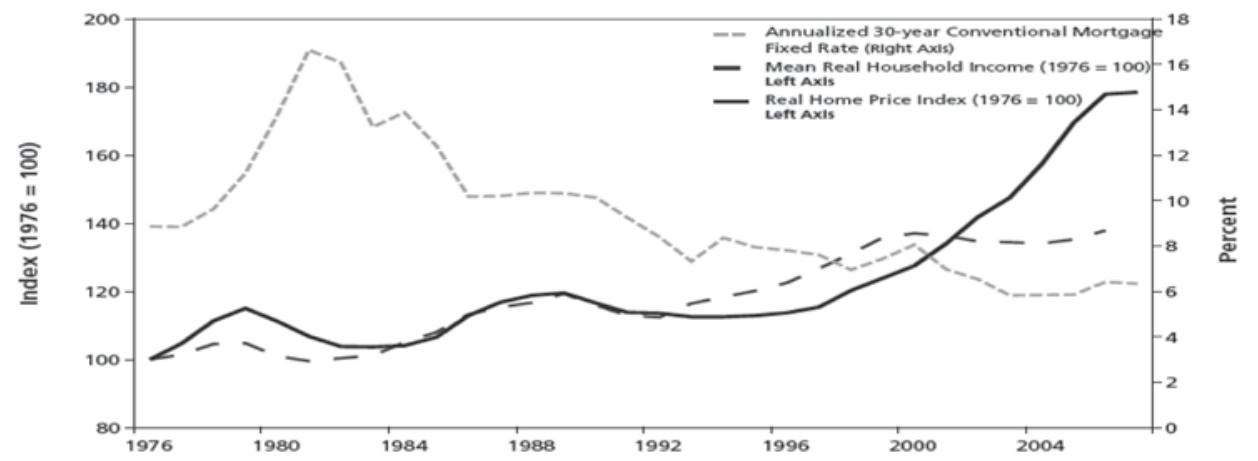

Source: OFHEO

1995 housing did increase faster than inflation, but not that much faster. After the mid 1990s, however, real house prices went on a sustained surge through 2005 making real estate a great investment opportunity. In 19952000 household income per capita rose substantially, contributing to the increase demand.

But what happened after is a constant surge in the housing prices from 1995 to the onwards. The increasing trend regardless of the constant decreasing household per capita income clearly shows a bubble in the housing market. In general experience of the other countries supports the view that the decline in mortgage interest rates was a key factor in triggering the run up of housing prices (Green and Wachter, 2007).

Was the Boom a Bubble?

In the aftermath of the housing boom, the question that economists are heatedly debating is how much of the increase in housing prices was due to economic fundamentals, and how much was due to a bubble-a rise in price due to "irrational exuberance" about future price appreciation (Alan Greenspan).

There were also reasons for housing prices to rise based on market fundamentals, however, such as rising incomes and falling mortgages rates (Getter et al., 2007). They put mainly two questions: first, why did bor- 
rowers increasingly use ARMs rather than locking in a relatively low fixed rate, which would have had no risk of future interest rate increases?; and second, why did mortgage lenders and investors not factor in rising rates when estimating the future probability of ARM delinquencies? Outcome of the results suggests that many borrowers might have been motivated by the prospect for short-term financial gains and investors turned to riskier types of MBs and these investments create a housing bubble which ultimately becomes the main reason of sub prime default.

\section{Lack of Transparency \& Accountability}

"Throughout the housing finance value chain, many participants contributed to the creation of bad mortgages and the selling of bad securities, apparently feeling secure that they would not be held accountable for their actions. A lender could sell exotic mortgages to home-owners, apparently without fear of repercussions if those mortgages failed. Similarly, a trader could sell toxic securities to investors, apparently without fear of personal responsibility if those contracts failed. And so it was for brokers, realtors, individuals in rating agencies, and other market participants, each maximizing his or her own gain and passing problems on down the line until the system itself collapsed. Because of the lack of participant accountability, the originate-to distribute model of mortgage finance, with its once great promise of managing risk, became itself a massive generator of risk."

Statement of John W. Snow before the Committee on Oversight and Government Reform United States House of Representatives

(October 23, 2008)

Former Fed Chairman Paul Volker has observed that problems of financial crisis began with a lack of accountability in mortgage lending and the trading of mortgage-backed securities. Financial executives spawned a proliferation of mortgage backed securities without integrity and traded them in non-transparent markets. According to (Larson, 2009) CEOs and Boards of Directors failed to be accountable to shareholders and to the public. They took on growing risk, ran reputable companies into the ground and paid themselves fat bonuses. Executives hide dangerously leveraged posi- 
tions from their shareholders and regulators by keeping risky transactions off Balance sheets and out of view

This attitude of individuals and even companies raises question of lack of transparency and accountability during the financial crisis. One of the essences of a well functioning free market is that the market itself holds players to account simply through who gets to sell their wares \& who does not. It appears that this market function has not helpful because Financial Crisis tells us the different story. According to Larson (2009), the German multinational firm Siemens recently agreed to fines of over $\$ 1.6$ billion to German and American authorities to resolve charges that it had systematically bribed public officials around the world in order to gain billions in government contracts. About one hundred U.S. firms were prosecuted by the Justice Department in 2008 for similar offences. Recently, Halliburton and Kellogg Brown \& Root agreed to pay $\$ 579$ million in fines related to bribes paid in Nigeria (Larson, 2009).

Federal Bureau of Investigation (FBI) has opened investigations into more than 500 cases of alleged corporate fraud, including 38 that involve important firms and are "directly related" to the national economic crisis. Deputy Director of FBI John Pistole told Congress that 38 companies are significantly large companies, everyone knows about them but he cannot comment publicly. In addition to major corporate fraud, Pistole testified that the number of mortgage fraud cases investigated by the FBI has risen from 881 in fiscal year 2006 to 1,600 in fiscal year 2008 (Jason, 2009).

According to Tatom (2008), the origins of the problem go back to 20042006, when a large share of new mortgage loans were made to subprime borrowers, borrowers who had relatively low credit scores and could not qualify for conventional mortgage loans at normal market interest rates. Many of these loans began to default much earlier than the normal experience from the past (Demyanyk, 2008). In fact, some of them went into default without ever making a payment.

Table 2 provides some statistics of mortgage origination. Annual originations grew from $\$ 2.2$ trillion in 2001 to nearly $\$ 4$ trillion in 2003 before settling around a figure of about $\$ 3$ trillion in the years 2004-2006. Of that, subprime originations grew from just $\$ 190$ billion in 2001 to $\$ 625$ billion in 2005; as a percent of the dollar value of total originations, subprimes grew from $8.6 \%$ to $20 \%$ of the market. Over the same period, the 
Table 2.

Mortgage Origination Statistics

\begin{tabular}{|l|c|c|c|c|c|}
\hline & $\begin{array}{c}\text { Totla Mortgage } \\
\text { Originations } \\
\text { (\$Billions) }\end{array}$ & $\begin{array}{c}\text { Sub Prime } \\
\text { Originations } \\
\text { (\$ Billions) }\end{array}$ & $\begin{array}{c}\text { Subprime share in } \\
\text { Total Originations \% } \\
\text { of \$ Value }\end{array}$ & $\begin{array}{c}\text { Subprime mortgage } \\
\text { Backed Securities } \\
\text { (\$ Billions) }\end{array}$ & $\begin{array}{c}\text { \% Subprimes } \\
\text { Securitized } \\
\text { (\% of dollar Value) }\end{array}$ \\
\hline 2001 & 2215 & 190 & 8.6 & 95 & 50.4 \\
2002 & 2885 & 231 & 8 & 121 & 52.7 \\
2003 & 3945 & 335 & 8.5 & 202 & 60.5 \\
2004 & 2920 & 540 & 18.5 & 401 & 74.3 \\
2005 & 3120 & 625 & 20 & 507 & 81.2 \\
2006 & 2980 & 600 & 20.1 & 483 & 80.5 \\
\hline
\end{tabular}

Source: Self-Made Table (Data Source: IMF).

percent of subprimes securitized increased from $50.4 \%$ to $80 \%$, which shows a growing trend of securitization.

Moreover, poor underwriting practices such as no down payments, no verification of income, assets, and jobs exacerbate the issue much. Over the past several years, the quantity and quality of loans across a variety of markets has weakened in two important ways. In terms of quantity, there was a large increase in lower-rated issuance from 2004 to 2007.

Figure 5 shows the quality of new debts issued from 1993 to 2007. Most of debts are low rated (B). From 2004 sudden increase in the issuance of low rated loans has been observed from as compared to the past years.

Quality wise we have seen increase in high combined loan-to-value. ${ }^{1}$ Figure 6 shows the issuance of loans with limited documentation. Starting

\footnotetext{
${ }^{1}$ Combined Loan to Value (ratio) (CLTV) is the proportion of loans (secured by a property) in relation to its value. The term "Combined Loan to Value" adds additional specificity to the basic Loan to Value, which simply indicates the ratio between one primary loan and the property value. When "Combined" is added, it indicates that additional loans on the property have been considered in the calculation of the percentage ratio. The aggregate principal balance(s) of all mortgages on a property divided by its appraised value or Purchase Price, whichever is less. Distinguishing CLTV from LTV serves to identify loan scenarios that involve more than one mortgage. For example, a property valued at $\$ 100,000$ with a single mortgage of $\$ 50,000$ has an LTV of $50 \%$. A similar property with a value of $\$ 100,000$ with a first mortgage of $\$ 50,000$ and a second mortgage of $\$ 25,000$ has an aggregate mortgage balance of $\$ 75,000$. The CLTV is 75 percent.
} 
Figure 5.

Quality of New Debt Issuance, 1993-2007

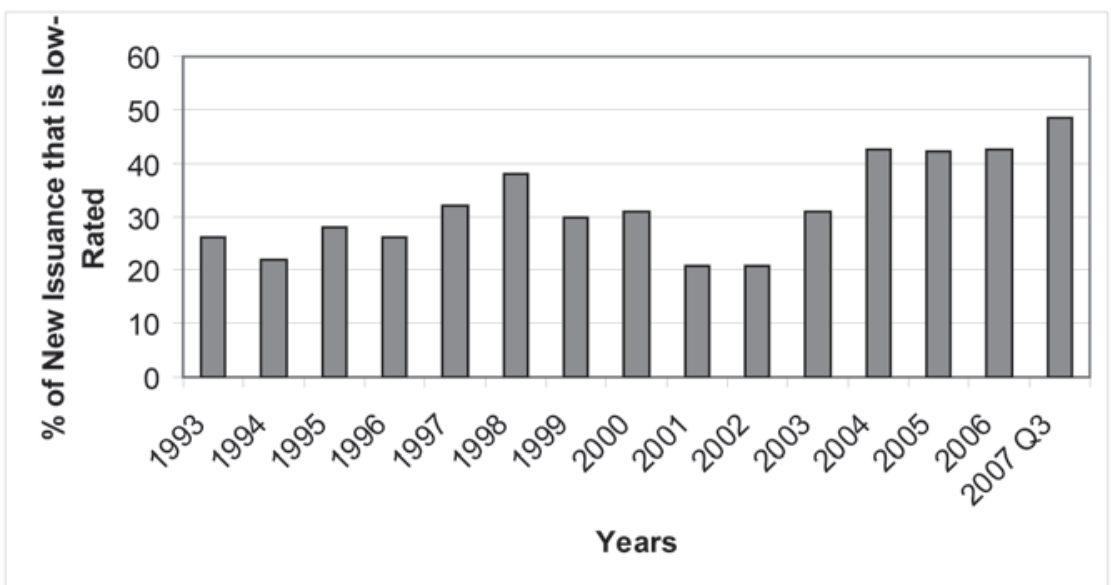

Source: Self-Made Figure (Data Source: Standard and Poor's).

Figure 6.

Combined loan to value

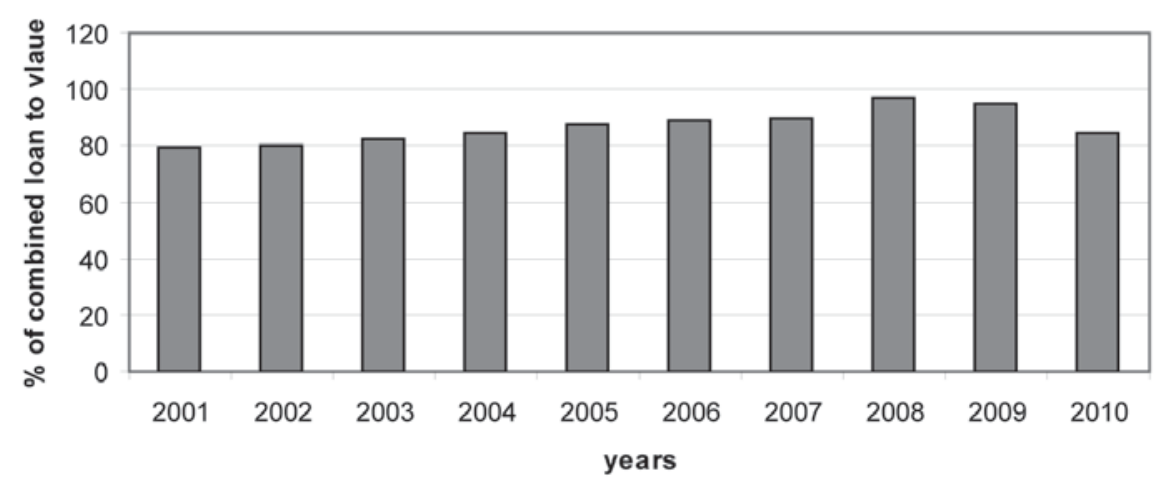

Source: Self-Made Figure (Data Source: Paulson and Company). 
from 2001 and going through 2006, it is visible from the graph that there were dramatic changes in the quantity of the loans during this period.

Table 3 shows the evolution of underwriting standards for subprime loans. The \%age of such loans with adjustable rates rose from $74 \%$ to $93 \%$ in the years 2001 to 2005 . Interest-only loans rose from zero to nearly $38 \%$ and the low or no doc share rose from $29 \%$ to more than $50 \%$. In other words, the riskiest types of subprimes ARMS and hybrid ARMS were favorites with securitizes. Debt payment to income ration has been increased from almost $40 \%$ to $43 \%$ while average loan to value ratio has been decreased.

Regardless of increase in low quality credit issuance and decrease in quantity there has been a parallel weakening of credit discipline in corporate credit markets, seen in the "flexing" of deals in favor of borrowers. Figure 7 below shows how credit risks have been increased by flex and reverses flex deals. From 2000 to 2002 we have seen terms flexed in favor of lenders while from 2003 to 2007 observed opposite.

In 2006 and 2007 a sharp increase has been seen in the volume of Covlite $^{2}$ or covenant lite loans. Cov-lite lending is seen as more risky because it

Table 3.

Underwriting Standards in Subprime Home-Purchase Loans

\begin{tabular}{|c|c|c|c|c|c|}
\hline Year & $\begin{array}{c}\text { ARM } \\
\text { Share }\end{array}$ & $\begin{array}{c}\text { Interest-Only } \\
\text { Arm Share }\end{array}$ & $\begin{array}{c}\text { Low-No- } \\
\text { Doc Share }\end{array}$ & $\begin{array}{c}\text { Debt Payment- } \\
\text { to-Income Ratio }\end{array}$ & $\begin{array}{c}\text { Average Loan-to- } \\
\text { Value Ratio }\end{array}$ \\
\hline 2001 & $73.80 \%$ & $0.00 \%$ & $28.50 \%$ & 39.7 & 84.04 \\
2002 & $80.00 \%$ & $2.30 \%$ & $38.60 \%$ & 40.1 & 84.42 \\
2003 & $80.10 \%$ & $8.60 \%$ & $42.80 \%$ & 40.5 & 86.09 \\
2004 & $89.40 \%$ & $27.20 \%$ & $45.20 \%$ & 41.2 & 84.86 \\
2005 & $93.30 \%$ & $37.80 \%$ & $50.70 \%$ & 41.8 & 83.24 \\
2006 & $91.30 \%$ & $22.80 \%$ & $50.80 \%$ & 42.4 & 83.35 \\
\hline
\end{tabular}

Source: Self-Made Figure (Data Source: IMF).

${ }^{2}$ Covenant lite is financial jargon for loan agreements which do not contain the usual protective covenants for the benefit of the lending party. It has been observed that cov-lite loans simply reflected changes in bargaining power between borrowers and lenders, and followed from the increased sophistication in the loans market where risk is quickly dispersed through syndication or credit derivatives. 
Figure 7.

Flex and Reverse Flex Deals

(in percentage of deals)

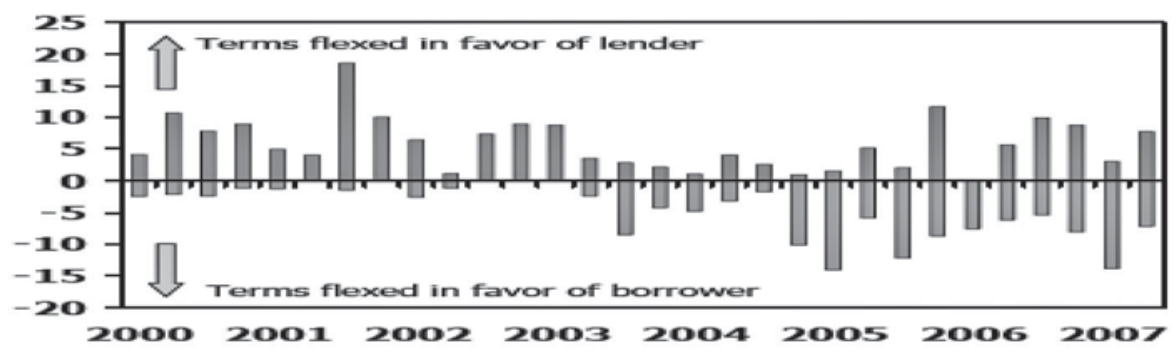

Source: IMF [http://www.imf.org/external/pubs/ft/fmu/eng/2007/charts.pdf].

Figure 8.

Volume of Covenant-Lite Loans

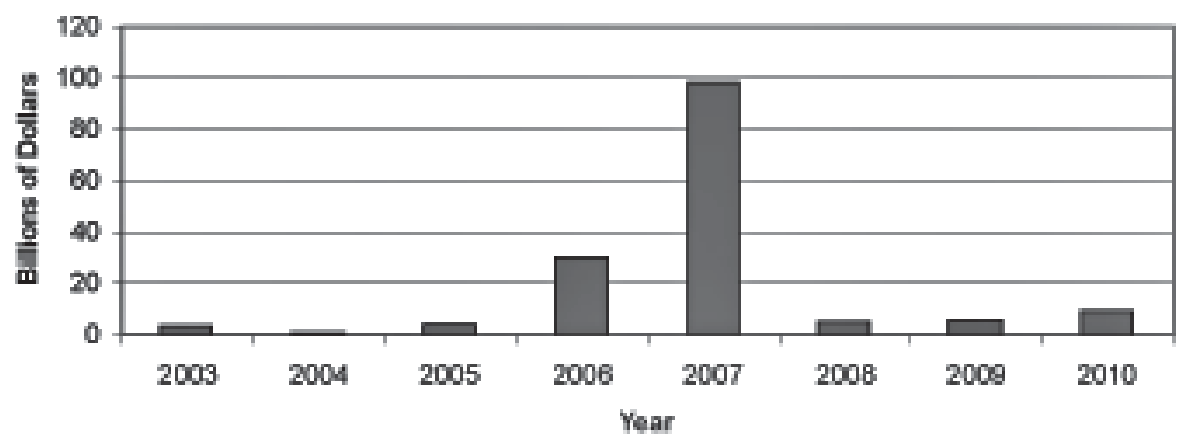

Source: Self-Made Figure (Data Source: ImF).

removes the early warning signs lenders would otherwise receive through traditional covenants. Figure 8 shows a tremendous increase in the use of Cov-Lite loans in the years 2006 and 2007. Especially in the year 2007 it cross the $\$ 100$ billion marks. Low credit quality and easy access to the credit made this possible. Firms looking for customers and eager to increase their share give loans even by compromising basic principles. Loans were granted on the minimum documentation possible and standard of documentation decreased. 
Figure 9.

Limited Documentation \%

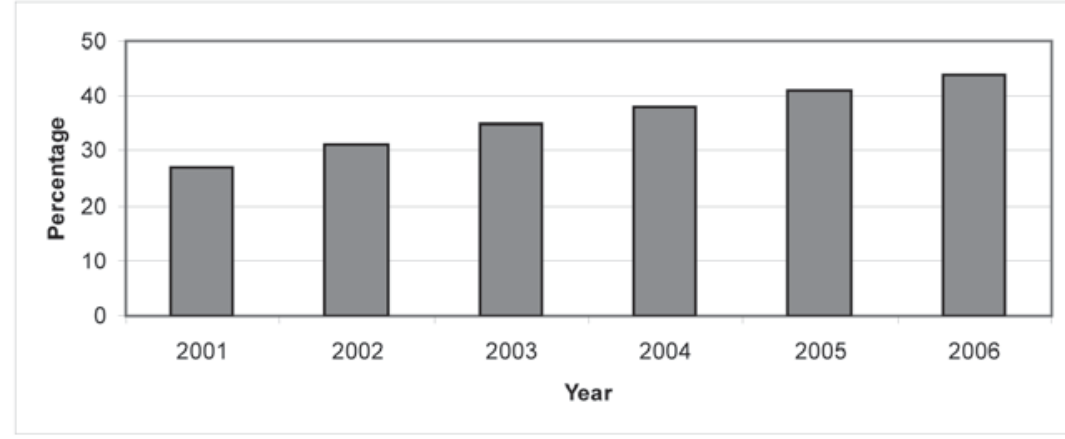

Source: Self-Made Figure (Data Source: Paulson and Company).

The Fed and other regulators generally supported new financial innovations. There may be some truth to both views. On the one hand, credit was widely available across all markets-mortgage, consumer, and corporate loans-with characteristics that suggested poorer and poorer loan quality. Figure 9 shows the loans which were issued during the period 2001 to 2007 with limited documentation. Percentage of limited documentation has increased from 27\% in 2001 to $44 \%$ in 2006.

One explanation for deteriorating loan quality is the huge growth in securitized credit. This is because the originate-to-distribute model of securitization reduces the incentives for the originator of the claims to monitor the creditworthiness of the borrower, because the originator has little or no skin in the game.

According to Jaffee (2008), securitization process has created a "moral hazard", allowing subprime lending risks to be passed in a sequence starting with mortgage brokers, then to lenders, then to securitizes, and ending as risks in investor portfolios. Although it is understandable that each of these transactors might participate in the chain as long as they were confident they could transfer the risk to the next stage.

Large quantity of risky loans with low quality creates troubles in credit markets. Loans were even given to those persons who don't afford it. It results in a mess in the credit market. Returns on these loans started to shrink and creates problems for the lenders. 
Figure 10 shows the subprime 60 days delinquency rate which is constantly rising from 2006 to 2008. Subprime delinquency has been increase from $0 \%$ in 2006 to alarming rate of almost 34 percent.

Lack of transparency and accountability in financial institutions motivated borrowers to borrow more and more even if they were not eligible.

Figure 10.

Subprime 60 days Delinquency Rate

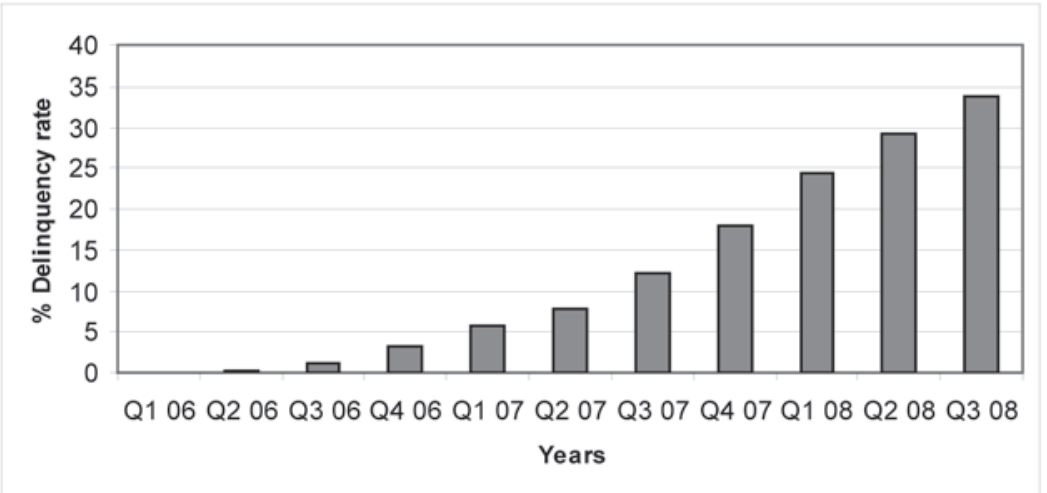

Source: Self-Made Figure (Data Source: Johnson and Company loan performance).

Figure 11.

Mortgage Fraud related SAR Financial Year 2004 to March 2009

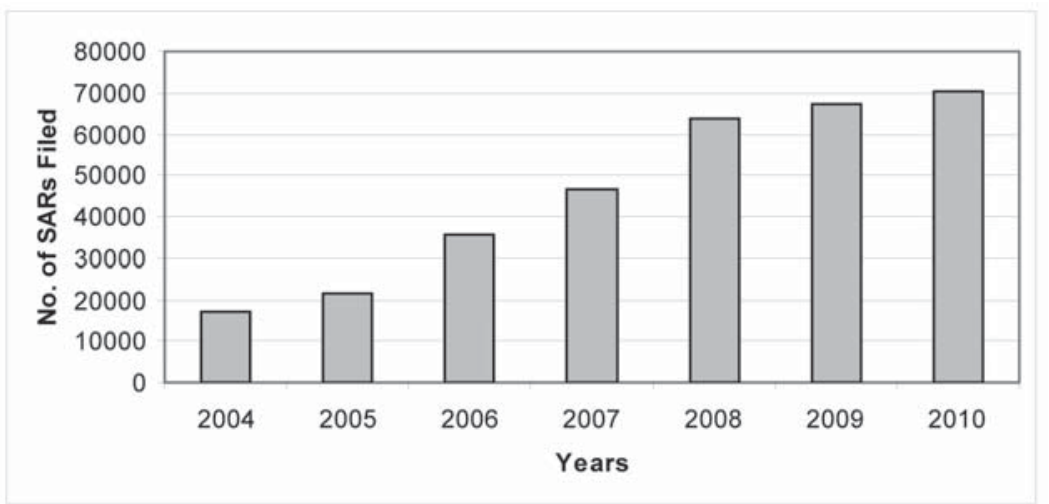

Source: Self-Made Figure (Data Source: FB 2008 Mortgage Fraud Report). 
Apart from the low standard of credit and minimum documentation there have been cases of massive frauds in mortgage loans. FBI issued a report on mortgage frauds in financial institutions. According to this report, Suspicious Activity Reports (SARs) from financial institutions indicate an increase in mortgage fraud reporting. There were 63,713 mortgage fraud related SARs filed in Financial Year 2008, a 36\% increase from Financial Year 2007. Figure 11 shows increasing trend of sars filed from the period 2004 to 2008.

In the same period, figure 12 shows SARs reported losses which are in \$Billions. SARs in FY2008 revealed losses of more than $\$ 1.4$ billion, an increase of $83.4 \%$ from FY2007. Additionally, SAR losses reported in the first six months of FY2009 exceed the same period in FY2008 by $\$ 208$ million.

FBI mortgage fraud investigations totaled 1,644 in FY2008, a 37\% increase from FY-2007 and a 100\% increase from FY-2006. Sixty-three percent $(1,035)$ of all pending FBI mortgage fraud investigations as of FY-2008 involved dollar losses of more than $\$ 1$ million. Figure 13 shows the story.

According to Crotty and Epstein (2008), main source of investment bank income has recently shifted from traditional activities such as advising on M\&As and bringing IPOs to market to fee income from securitization and trading on their own account. Much of the trading is in mortgage-backed securities, which they create and both sell to others and hold in their own trading accounts.

Figure 12.

Mortgage Fraud SAR losses (\$ Millions) from 2004 to March 2009

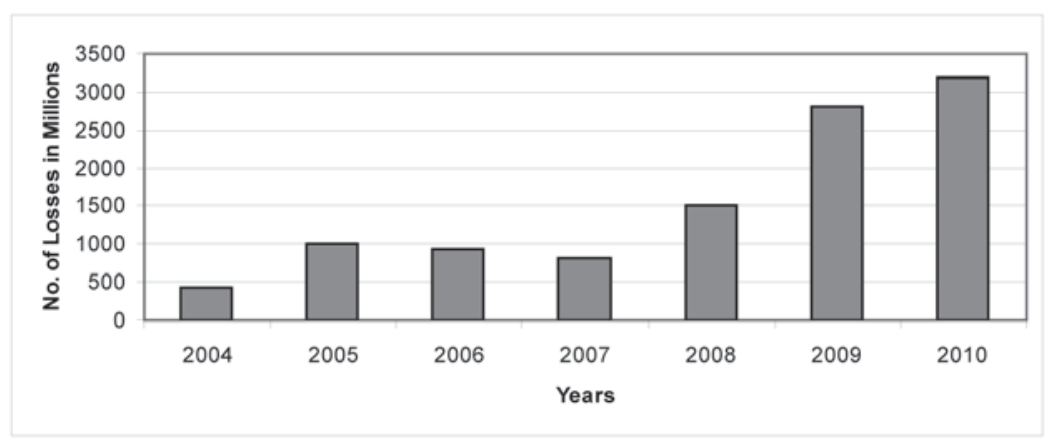

Source: Self-Made Figure (Data Source: FB 2008 Mortgage Fraud Report). 
Figure 13.

Increase in FBI Mortgage Fraud Pending Investigations

from 2004 to March 2009

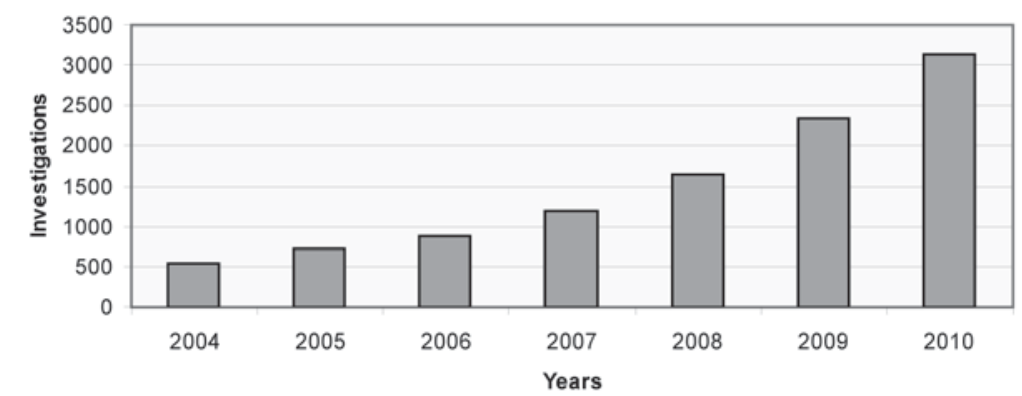

Source: Self-Made Figure (Data Source: FBI 2008 Mortgage Fraud Report).

\section{Conclusions}

In the whole discussion we have proved that there was a bubble in the Housing Market and when the bubble burst it generated the Crisis. Easy availability of mortgage loans with low documentation and almost zero interest rates also attract those people to mortgage houses who cannot afford it. This results in Foreclosures and Delinquencies.

From figures 1 to 4 we have proved through different aspects how the bubble was created and then after that how it burst. Specially the figure one tabulate the data from 1955 to 2010 . The data showed a high increase in ratio of home prices to up to more than $14 \%$ which was the historically high and then in the next five year it dropped suddenly to normal level. All these things show a definite bubble in the market.

There were serious transparency issues in the mortgage market, like Citigroup -one of the biggest players in the mortgage securitization- frenzy having global worth $\$ 3.8$ trillion at their peak in $2006,11 \%$ higher than in the super year of 2000 .

These profits with high risk strategies enabled the firms to reward its executives. Top traders and executives receive sky high bonuses in years in which risk-taking behavior generates high profits. In 2006, Goldman Sachs' bonus pool totaled $\$ 16$ billion. Top executives of Wall Street received bonuses up to $\$ 50$ million that year. Five largest investment banks 
-Merrill, Goldman Sach, Morgan Stanley, Lehman Brothers and Bear Stearns paid out about $\$ 66$ billion in compensation in 2007 , including an estimated $\$ 40$ billion in bonuses. Despite the decline in profit the bonus figure was higher than the $\$ 36$ billion last year.

With the help of FIA investigation we have proved that the number of suspicious activities have been increased in the last five years. There were alarming situation: specially the flex and reverse flex deals clearly shows the situation in favor of the borrower. These severe lacks of transparency and accountability practices generate the mess in which we are now. Strange part of the story is that still no proper accountability has been fixed on any one. For transparency and accountability in future US Govt. has taken two steps. First, Financial Stability Plan has been constituted with the purpose of "protect taxpayers and ensure that every dollar is directed toward lending and economic revitalization". Second congress has passed Commission on Financial Crisis Accountability Act 2009.

It is highly recommended that the whole system of mortgage should be checked and reforms should be made on the large level. Especially the Commission's recommendations should be implemented with true letter and spirit. There should be check on the bonuses to the executives of the financial institutions and there should be a law to restrict them from taking too much risk.

\section{References}

Baily Martin Neil, Robert E. Litan, and Matthew S. Johnson (2008), “The Origins of the Financial Crisis", Brooking Institution, working paper no. 1519,

Case, Karl and Shiller (2003), "Is There a Bubble Housing Market?", Brookings Papers on Economic Activity, no. 2, pp. 299-342.

Cecchetti Stephen (2008), "Monetary Policy and the Financial Crisis of 20072008”, Journal of Economic Theory, no. 92, pp. 1-34.

Crotty James and Gerald Epstein (2008), "Proposals for Effectively Regulating the U.S. Financial System to Avoid Yet another Meltdown", Economist, $15^{\text {th }}$ January, 2008.

Dymyanyk Yuliya, Hemert (2008), "Understanding the Subprime Mortgage Crisis", Financial Times, 11-02-2009.

Getter et al. (2007), "Financial Crisis? The Liquidity Crunch of August 2007", National Bureau of Economic Research, working paper, no. 3927, Cambridge, Mass. Cambridge Press, pp. 1-38. 
Green, Richard and Susan Wachter (2007), “The Housing Finance Revolution”, Paper presented at the Federal Reserve Bank of Kansas City Symposium.

Jaffee Dwight (2008), "The U.S. Subprime Mortgage Crisis: Issues Raised and Lessons Learned", The Economist, 15-12-2009

Jason (2009), "A Decomposition Analysis of Regional Poverty in Russia”, Financial Times, 21-10-2008

Kregel, J. (2008), Minsky's Cushions of Safety. Systemic Risk and the Crisis in the U.S. Subprime Mortgage Market, The Levy Economics Institute of Bard College, Public Policy Brief, no. 93, http://www.levy.org/pubs/ppb_93.pdf

Larson, Alan (2009), "Restoring Trust through Accountability, Transparency and Integrity: The Key Ingredient for Recovery", Financial Times, 25-05-2009.

Magnus (2007), "Subprime Mortgage Lending and the Capital Markets" [Online], San Francisco, FRBSF. Available from: http://www.frbsf.org/publications/ economics/letter/2001/el2001-38.pdf [Accessed 23 July 2007].

McCauley (2008), "How 'Credit Default Swaps' —an Insurance against Bad Loans-Turned from a Smart Tet into a Killer", News Week, September 27.

Minsky, H. P. (1986), The Financial Instability Hypothesis, The Levy Economics Institute of Working Paper no. 74.

Portes, R. and D. Vines (1997), Coping with Capital Inflows, Commonwealth Secretariat, London, Economic Paper no. 30.

Shostak, Anthony (2008), "Inequality Decomposition by Factor Components", Econometrica, vol. 50, no. 1, Chicago, MIT-Press, pp. 193-211.

Tatom, John (2008), “Two Pronged Assault on us Economy”, Daily Telegraph, 2812-2008.

Taylor, John B. (2008), Getting off Track: How Govt. Actions and Interventions Caused, Prolonged and Worsened the Financial Crisis, Hoover Institution Press, USA.

Whalen, C. (2008), "A Minsky Moment: Reflections on Hyman P. Minsky (19191996)", Journal of Economic Issues, vol. 42, no. 1.

Wolfson (1994), "The Aggregate Demand for Treasury Debt", working paper, North-western University; (2008) Economics, vol. 9, no. 1, New Jersey, Blackwell Publishing, pp. 25-46.

Wray, R. L. (2008), "Financial Markets Meltdown. What can we Learn from Minsky?”, Public Policy Brief, no. 94, The Levy Economics of Bard College. and B. D. Papadimitriou (1999), "Minsky's Analysis of Financial Capitalism", Working Paper no. 275, The Jerome Levy Economics Institute.

Artículo recibido el 5 de septiembre de 2011

Segunda versión recibida el 17 de mayo de 2012

Artículo aprobado el 19 de junio de 2012 



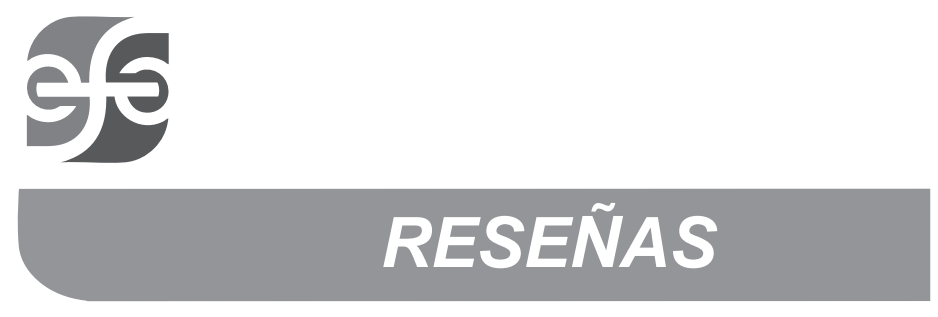


